\title{
The effects of elaboration on recognition memory
}

\author{
RONALD P. FISHER and FERGUS I. M. CRAIK \\ Erindale College, University of Toronto, Toronto, Ontario L5L 1C6, Canada
}

\begin{abstract}
Two experiments were conducted to examine the effects of elaboration on recognition memory. Subjects were given either simple or complex sentences to learn and were tested for recognition of either an individual target word or the entire sentence. Complex sentences supported better recognition performance only when the test item allowed the subject to easily redintegrate the initial encoding context, either by re-presenting the encoded sentence as the test item or by constructing sentences such that the component words of the sentence could be easily redintegrated from an individual target item. It was suggested that complex, elaborate encoding established a richer trace, but that this richness can be utilized to enhance recognition only when the test conditions permit a reinstatement of the original encoding context.
\end{abstract}

One major factor affecting retention of verbal material appears to be the degree of elaboration conferred on the material by the encoding environment. As the term is used here, elaboration refers to the richness or extensiveness of encoding within a specific domain; several recent studies have shown that increased elaboration is associated with higher levels of retention (Anderson \& Reder, 1979; Craik \& Tulving, 1975; Eysenck, 1979; Klein \& Saltz, 1976). Speculatively, elaborate encodings may be more distinctive and thus more discriminable from other encodings in the memory system, and it has been suggested that distinctiveness is the crucial factor underlying the effect of elaboration on memory (Eysenck, 1979; Jacoby \& Craik, 1979; Klein \& Saltz, 1976). That is, a greater degree of elaboration results in a more distinctive encoding, and, provided the retrieval environment is compatible (Fisher \& Craik, 1977; Morris, Bransford, \& Franks, 1977), the enhanced distinctiveness facilitates retrieval. In one relevant study, Craik and Tulving (1975, Experiment 7) embedded words in sentence frames that were either "simple" (e.g., I lost my WATCH), "medium" (e.g., The villain had a KNIFE in his pocket), or "complex" (e.g., The BULL was standing in deep grass by the edge of the field). It was assumed that the embedded target words (capitalized in the above examples) were elaborated to a progressively greater degree from simple to complex contexts, and, in line with the notion that elaboration is related positively to retention, Craik and Tulving found that free recall of the embedded words increased monotonically from simple to complex cases. When the sentence frames were provided as cues at retrieval,

This research was supported by National Research Council of Canada Grant A8261 to Fergus I. M. Craik. Requests for reprints should be sent to Ronald Fisher, Department of Psychology, Florida International University, Tamiami Campus, Miami, Florida 33199. recall of the embedded words also rose from simple to complex, but more steeply than in the case of free recall. Thus, elaborating words to various degrees by increasing the richness and complexity of the sentence context systematically increases the levels of free and cued recall.

The starting point of the present investigation was a pilot study in which the sentence-complexity manipulation was repeated, but with recognition as the retention test. Tulving (Note 1) had run such a study as a laboratory exercise and had found no effect of sentence complexity on recognition. In light of the free and cued recall findings, the null effect for recogniton seemed surprising, so our pilot study was undertaken to explore the situation further. Elaboration was manipulated by varying the length and complexity of the sentence frames in which the target words were embedded. For each of 40 high-frequency noun targets, there were two sentence frames: simple and complex. The surface structure of the simple sentences was either subject-verb or subjectverb-object (e.g., The recipe called for sugar), whereas the complex sentences contained additional word or phrase modifiers before the subject or object (e.g., The exotic recipe called for 4 level cups of sugar). The target word, which was later tested, was always the subject or object of the sentence and was always preceded by a modifier in the complex sentence. Forty sentences, alternating between simple and complex, were read aloud to each of 16 subjects, who were told to try to remember as much as they could about the sentences for a later test. There was no indication about the nature of the test. Immediately after the final sentence, the subjects were given two batch-recognition tests, one in which the 20 simple words were mixed with 30 foil words and another in which 20 complex words were mixed with 30 foils. Test order, assignment of words to sentence type, and assignment of foils to conditions 
were counterbalanced across subjects. Subjects were asked to circle the words they had heard in the sentences and were free to circle as many as they wished.

The mean $\mathrm{d}^{\prime}$ scores for words embedded in simple and complex sentences were 1.59 and 1.49 , respectively. These scores did not differ reliably $[F(1,15)<1]$. Thus, Tulving's (Note 1) observation that differences in sentence complexity do not affect recognition memory for the embedded target words was confirmed.

Why should sentence complexity affect recall but not recognition? One possibility is that recall and recognition depend on qualitatively different subprocesses (Kintsch, 1970; McCormack, 1972) and are therefore differentially responsive to some variables. However, given the arguments by Lockhart, Craik, and Jacoby (1976) and Tulving (1976) stressing the similarity between recall and recognition, and given the recent evidence presented by Fisher (1979) and Rabinowitz, Mandler, and Patterson (1977) suggesting the presence of retrieval operations in both recall and recognition, we were led to test a second possible explanation of the present data. This alternative possibility is that both recall and recognition are responsive to sentence-complexity manipulations, but that the type of recognition test used in the pilot study was inappropriate to observe these effects. The notion of an "appropriate" test situation has recently been stressed by Morris et al. (1977). They suggested that the demonstrated effectiveness of a particular encoding activity depends on the nature of the test situation. To illustrate their argument, Morris et al. showed that, contrary to the usual finding, rhyme encoding was associated with higher levels of retention than was semantic encoding, provided that the test situation involved a test for phonemic information. Similar points have also been made by McDaniel, Friedman, and Bourne (1978) and by Tulving (1979).

The failure to find reliable effects of sentence complexity in the pilot study may reflect a similar phenomenon: The complex sentence frames may have induced richer traces than did simple sentence frames, but the retrieval cues may not have been sufficiently similar to the encoding context to utilize this greater potential. That is, by providing only the individual target words as "cues" at retrieval, the richness of the sentence frames may not have been reinstated. If this is so, we should expect that if the retrieval cue were more similar to the original sentence frame, the effect of sentence complexity would reappear. In the recall data reported by Craik and Tulving (1975, Experiment 7), it was found that the advantage of complex encoding was much larger when the encoding context (sentence frame) was incorporated in the retrieval cue (i.e., cued recall) than when it was absent (free recall). We might therefore expect that if the encoding context were incorporated in the retrieval cue in a recognition test, events encoded in a complex fashion would be better recognized than events encoded in a simple fashion. In order to test this hypothesis in Experiment 1, we presented words in the context of either simple or complex sentence frames and tested for recognition of either the individual words or the entire sentences. Our expectation was that the effects of encoding complexity would manifest themselves when the complexity was incorporated in the retrieval cue, that is, when the test item was the entire sentence, but not when the retrieval cue failed to incorporate this complexity, that is, when individual words were presented for recognition.

\section{EXPERIMENT 1}

\section{Method}

Subjects and Stimuli. Thirty-two Florida International University students of both sexes were auditorily presented with a series of sentences and were tested for recognition of either individual words that appeared in the sentences or the entire sentences. The sentences were identical to those used in the pilot study.

Procedure. Subjects were tested in small groups of one to four. They were presented a series of 76 simple and complex sentences, of which the middle 40 sentences ( 20 simple and 20 complex) were tested later. The sentences were read aloud by the experimenter in a normal speaking voice, with a 3-sec delay between sentences. The subjects were told to listen to the sentences and to try to remember as much as possible about them. Immediately after the final sentence, half the subjects were given a recognition test for the target words alone and half were tested for recognition of the entire sentences. In the wordalone group, the subjects were given two batch-recognition test sheets, each containing 50 words: Twenty of these had appeared in the presented sentences, and 30 were distractors. All subjects in this group performed both recognition tests, one for target words appearing in simple sentences and one for words appearing in complex sentences. In the sentence group, the subjects were given two batch-recognition test sheets, each containing 50 sentences. One test consisted of simple sentences (20 "old" sentences plus 30 distractors), and the other consisted of complex sentences ( 20 "old" plus 30 distractors). For both simple and complex tests, the distractor sentences were similar in structure to the target sentences: Mean word length and number of modifying words or phrases were approximately the same for targets and distractors. The subjects were allowed unlimited time and were free to circle fewer or more than 20 alternatives for all tests.

Design. Sentence complexity was manipulated within subjects, and type of test was manipulated between groups. For each of the test conditions, assignment of words to sentence type and test order (simple first or complex first) were counterbalanced across subjects.

\section{Results and Discussion}

Table 1 shows hit rates, false alarm rates, and $\mathrm{d}^{\prime}$ scores for each condition. The scores show that words

\section{Table 1}

Hit Rates (H), False Alarm Rates (FA), and d' Scores for Simple and Complex Sentences in Experiment 1

\begin{tabular}{|c|c|c|c|c|c|c|}
\hline \multirow{3}{*}{$\begin{array}{c}\text { Test } \\
\text { Condition }\end{array}$} & \multicolumn{6}{|c|}{ Sentence Type } \\
\hline & \multicolumn{3}{|c|}{ Simple } & \multicolumn{3}{|c|}{ Complex } \\
\hline & $\mathbf{H}$ & FA & $\mathrm{d}^{\prime}$ & $\mathbf{H}$ & FA & $\mathrm{d}^{\prime}$ \\
\hline Word Alone & .53 & .13 & 1.61 & .51 & .15 & 1.54 \\
\hline Sentence & .46 & .08 & 1.75 & .63 & .04 & 2.46 \\
\hline
\end{tabular}


encoded in complex sentences were recognized better than were words encoded in simple sentences. However, this result held only for the sentence test condition; in the word-alone condition, sentence complexity was again found to have no effect on recognition scores. Since $d^{\prime}$ scores give the most valid index of performance levels in this case, an analysis of variance was carried out on these scores. The analysis showed a reliable effect of sentence complexity $[\mathrm{F}(1,30)=5.20$, MSe $=$ $.316, p<.01]$, a reliable effect of test type $[F(1,30)=$ 10.26 , MSe $=.437, p<.01]$, and a reliable interaction between sentence complexity and type of test $[F(1,30)$ $=7.84, \mathrm{MSe}=.316, \mathrm{p}<.01]$.

The observation that sentence complexity had reliable effects on sentence but not on word-alone recognition performance suggests that the null effect of sentence complexity obtained in the pilot study is not an inevitable feature of recognition testing. It seems, rather, that beneficial effects of sentence complexity on recognition may be observed only when retrieval conditions lead to reinstatement of the initial elaborate encoding context. Plausibly, when the test word was presented alone in the pilot study and in the present experiment, the recognition decision was made without redintegration of the original sentence context, and thus no beneficial effect of sentence complexity was observed. In line with notions of appropriate test situations (Morris et al., 1977; Tulving, 1979), an elaborate encoding environment does not appear to enhance recognition memory unless retrieval conditions induce reinstatement of the original elaborate environment. The failure of sentence complexity to affect recognition in the word-alone condition may thus be analogous to the well-known phenomenon of failure to recognize the librarian or the butcher when he or she is encountered in atypical surroundings; the face alone does not provide sufficient information to redintegrate the initial context, just as the word alone does not provide sufficient information to redintegrate the original sentence.

A second experiment was conducted to test the reinstatement notion directly. Sentences were constructed in which the potential recognition target words were more or less likely to redintegrate the rest of the sentence. If sentence complexity aids recognition only when the initial context is either reprovided or redintegrated at retrieval, it is expected that complexity will enhance recognition in the high-redintegration case, but not in the low-redintegration case. Experiment 2 might also serve to eliminate an alternative explanation of the results of Experiment 1: Since the sentence recognition test involves a re-presentation of the encoding sentence as the test item and complex sentences contained more words than did simple sentences, there were more words in the complex test sentences than in the simple test sentences. Therefore, if the subject made an "old" response on the basis of having recognized several words in the test sentence, he might have performed better on the complex sentences only because there were more potentially recognizable words. The Complexity by Type of Test interaction might then be simply a function of the number of words in the test item.

\section{EXPERIMENT 2}

\section{Method}

Materials. As in Experiment 1, there were simple and complex versions for each of $\mathbf{4 0}$ high-frequency noun targets (to be tested later), with the target word being either the subject or the object of the sentence. The sentences were constructed so that for both the simple and complex sentences, half were easily redintegrated from the target word (high redintegration) and half were difficult to redintegrate from the target word (low redintegration). This was accomplished by selecting a series of target words and then constructing sentence frames around each word such that either several of the content words of the sentence frames were high associates of the target word or none of the content words was a high associate of the target word. For highredintegration complex sentences, at least three of the content words in the sentence frames were high associates of the target word; for high-redintegration simple sentences, at least one of the content words was a high associate. For both types of lowredintegration sentences, simple and complex, none of the content words was a high associate of the target word. Examples of the four types of sentences are as follows (italicized words are high associates of the capitalized target words): highredintegration simple-He washed in the BATH; lowredintegration simple-He took a BATH; high-redintegration complex-He felt clean after washing in a hot BATH; lowredintegration complex-He felt refreshed after taking his morning BATH.

Subjects, Procedure, and Design. The experiment was conducted as part of a demonstration at the Ontario Science Centre in Toronto. The 32 volunteer subjects participating in this study ranged in age from approximately 15 to 50 years. All subjects were tested in small groups of 2-10.

After a brief introduction, in which the subjects were told that they would hear a series of unrelated sentences and that they should try to remember as many of these as possible, the experimenter read aloud 76 sentences. The middle 40, which were tested later, were evenly distributed among the four sentence types, formed by the crossing of redintegration (high vs. low) and complexity (simple vs. complex). Following the last sentence, all subjects received four separate recognition tests. On each of the four tests, there appeared 25 individual words, 10 of which had appeared in the sentences that the subjects previously heard. The subjects were asked to circle these words but were free to circle fewer or more than 10 if they desired. Test order, assignment of words to sentence type, and assignment of distractors to the four conditions were counterbalanced across subjects.

\section{Results}

Hit rates, false alarm rates, and $\mathrm{d}^{\prime}$ values for the four conditions are presented in Table 2 . It is clear that sentence complexity confers no advantage to word recognition in the low-redintegration condition, but that such an advantage is strongly present in the highredintegration condition. These observations were supported by an analysis of variance on the $d^{\prime}$ data, in which the only reliable sources of variation were sentence complexity $[\mathrm{F}(1,31)=7.55, \quad \mathrm{MSe}=.389$, 
Table 2

Hit Rates (H), False Alarm Rates (FA), and d' Scores for Simple and Complex Sentences in Experiment 2

\begin{tabular}{cccccccc} 
& \multicolumn{4}{c}{ Sentence Type } \\
\cline { 2 - 4 } \cline { 5 - 7 } $\begin{array}{c}\text { Redinte- } \\
\text { gration }\end{array}$ & $\mathrm{H}$ & FA & $\mathrm{d}^{\prime}$ & & $\mathrm{H}$ & FA & $\mathrm{d}^{\prime}$ \\
\hline Low & .54 & .20 & 1.50 & .57 & .21 & 1.50 \\
High & .58 & .22 & 1.42 & .67 & .15 & 2.02 \\
\hline
\end{tabular}

$\mathrm{p}<.05]$ and the interaction between complexity and redintegration $[\mathrm{F}(1,31)=7.37, \mathrm{MSe}=.374, \mathrm{p}<.05]$.

\section{Discussion}

The general trend observed in Experiment 1 with type of test as the parameter (that is, greater superiority of complex encoding under sentence than under wordalone conditions) was observed in Experiment 2, with ease of redintegration as the parameter (that is, greater superiority of complex encoding with high- than with low-redintegration sentences). The similarity in the pattern of results between the two experiments supports the notion that the more complex encoding context must be reinstated at retrieval before the greater complexity enhances recognition memory performance. When the retrieval cue (in this case the target word presented for recognition) did not lead easily to the reinstatement of the original sentence frame (low redintegration), there was no effect of sentence structure. Only in the condition that permitted relatively easy reinstatement of the original encoding context from the retrieval cue (high redintegration) did subjects benefit from the complexity of sentence structure.

\section{GENERAL DISCUSSION}

The present series of studies increases our knowledge of the circumstances under which elaboration of an event at encoding acts to enhance later memory of the event. Craik and Tulving (1975) showed that words placed in the context of complex sentences were better recalled than were words embedded in simple sentences; their explanation was that the complex sentence frame acted to elaborate the target word and that such elaboration was beneficial to recall. The present experiments have shown that this conclusion can be extended to recognition memory, but only under specific test conditions: namely, those that permit reinstatement of the original elaborate encoding context. When the target event is re-presented for recognition under conditions that do not allow for easy reinstatement of the encoding context, elaboration of the context has little or no effect (Experiment 1, word-alone condition; Experiment 2, lowredintegration condition). Elaboration of the context does have a beneficial effect on recognition, however, under retrieval conditions that reprovide the original context or allow for its reconstruction (Experiment 1, sentence condition; Experiment 2, high-redintegration condition). Interestingly, when Craik and Tulving's recall data are reexamined, they also show strong effects of reinstatement of initial context: Under free recall conditions, the effect of sentence complexity is quite small, but the effect is greatly enhanced by reproviding the sentence frames in cued recall. These parallel findings between recall and recognition support the position that common processes underlie both forms of memory (Fisher, 1979; Lockhart et al., 1976; Rabinowitz et al., 1977).

The present results suggest that a modification is necessary in the way that the effects of elaboration are conceptualized. We had initially assumed that complex sentence frames have their beneficial effect on retention by elaborating the target word itself, that is, that an enriched version of the target word was encoded when embedded in a complex sentence. The finding that recognition of target words alone was not enhanced by sentence complexity (pilot study and Experiment 1) now makes this assumption quite unlikely. Rather, it seems that the elaboration in question consists of the total unit of word plus sentence frame; the whole unit must be retrieved at test before the beneficial effects of complexity are observed. It follows from this analysis that the effects of elaboration will be found with single items only in cases in which the item and its elaborating context are well integrated, and therefore presentation of the item alone will serve to redintegrate the encoding context. Thus, faces of well-known people are recognized even out of context, whereas faces of people we know more casually are not recognized (or only partially recognized) wher they are encountered in an unfamiliar setting.

In terms of the experimental literature, the present results are reminiscent of the findings of Winograd, Karchmer, and Russell (1971), who showed that instructions to form an image from pairs of words enhanced later recognition of one member of the pair only when the other member of the pair was also present during the recognition test. The present results are also compatible with the demonstration of context effects in recognition memory by Thomson (1972) and with the encoding-specificity principle of Tulving and Thomson (1973).

It thus appears that the mere addition of elaborative information at encoding is not sufficient to improve retention. Rather, it alters only the potential memorability of an event, and reinstatement of the initial encoding context by one means or another is also necessary before the beneficial effects are observed. Some recent attempts to describe the conditions under which elaboration will and will not affect performance 
have been made by Anderson and Reder (1979), Bransford, Franks, Morris, and Stein (1979), and Eysenck (1979). Anderson and Reder and Bransford et al. have suggested that the effectiveness of an encoding elaboration will depend upon the relation between the type of elaboration required and the current state of the learner's knowledge: Elaborations that make use of well-practiced skills support better retention than do those that use poorly learned skills. Second, as suggested by Bransford et al., Eysenck, and the present results, the effectiveness of an encoding elaboration will be moderated by the requirements of the test condition: Elaborate encoding will be effective to the extent that the encoding context is reinstated by the retrieval context. It appears to us that the current approach of identifying those conditions under which elaboration does and does not facilitate performance represents a healthy complication of the original question of whether or not elaboration affects performance.

\section{REFERENCE NOTE}

1. Tulving, E. Personal communication, June 1977.

\section{REFERENCES}

Anderson, J. R., \& Reder, L. M. An elaborative processing explanation of depth of processing. In L. S. Cermak \& F. I. M. Craik (Eds.), Levels of processing in human memory. Hillsdale, N.J: Erlbaum, 1979.

Bransford, J. D., Franks, J. J., Morris, C. D., \& Stein, B. S. Some general constraints on learning and memory research. In L. S. Cermak \& F. I. M. Craik (Eds.), Levels of processing in human memory. Hillsdale, N.J: Erlbaum, 1979.

Craik, F. I. M., \& Tulving, E. Depth of processing and the retention of words in episodic memory. Journal of Experimental Psychology: General, 1975, 104, 268-294.

EySENCK, M. W. Depth, elaboration, and distinctiveness. In L. S. Cermak \& F. I. M. Craik (Eds.), Levels of processing in human memory. Hillsdale, N.J: Erlbaum, 1979.
Fishe R, R. P. Retrieval operations in cued recall and recognition. Memory \& Cognition, 1979, 7, 224-231.

Fisher, R. P., \& Craik, F. I. M. Interaction between encoding and retrieval operations in cued recall. Journal of Experimental Psychology: Human Learning and Memory, 1977, 3, 701-711.

JaCoBY, L. L., \& Craik, F. I. M. Effects of elaboration of encoding and retrieval: Trace distinctiveness and recovery of initial context. In L. S. Cermak \& F. I. M. Craik (Eds.), Levels of processing in human memory. Hillsdale, N.J: Erlbaum, 1979.

KINTSCH, W. Learning, memory, and conceptual processes. New York: Wiley, 1970.

KLE IN, K., \& SAltz, E. Specifying the mechanisms in a levels-ofprocessing approach to memory. Journal of Experimental Psychology: Human Learning and Memory, 1976, 2, 671-679.

Lockhart, R. S., Craik, F. I. M., \& Jacoby, L. L. Depth of processing in recognition and recall: Some aspects of a general memory system. In J. Brown (Ed.), Recognition and recall. London: Wiley, 1976.

MCCormack, P. D. Recognition memory: How complex a retrieval system? Canadian Journal of Psychology, 1972, 26, 18-41.

McDaniel, M. A., Friedman, A., \& Bourne, L. E., Jr. Remembering the levels of information in words. Memory \& Cognition, 1978, 6, 156-164.

Morris, C. D., Bransford, J. D., \& Franks, J. J. Levels of processing versus transfer appropriate processing. Journal of Verbal Learning and Verbal Behavior, 1977, 16, 519-533.

Rabinowitz, J. C., Mandler, G., \& Patterson, K. E. Determinants of recognition and recall: Accessibility and generation. Journal of Experimental Psychology: General, 1977, 106, 302-329.

Thomson, D. M. Context effects in recognition memory. Journal of Verbal Learning and Verbal Behavior, 1972, 11, 497-511.

Tulving, E. Ecphoric processes in recall and recognition. In J. Brown (Ed.), Recognition and recall. London: Wiley, 1976.

Tulving, E. Relation between encoding specificity and levels of processing. In L. S. Cermak \& F. I. M. Craik (Eds.), Levels of processing in human memory. Hillsdale, N.J: Erlbaum, 1979.

Tulving, E., \& Thomson, D. M. Encoding specificity and retrieval processes in episodic memory. Psychological Review, 1973, 80, 352-373.

Winograd, E., Karchmer, M. A., \& Russell, I. S. Role of encoding unitization in cued recognition memory. Journal of Verbal Learning and Verbal Behavior, 1971, 10, 199-206.

(Received for publication February 19, 1980; revision accepted March 19, 1980.) 